\title{
Sound Generation in the Outlet Section of Gas Turbine Combustion Chambers
}

\author{
C. Schemel ${ }^{*}$ and F. Thiele ${ }^{\dagger}$ \\ Berlin University of Technology, Hermann-Föttinger-Institute of Fluid Mechanics \\ and \\ F. Bake ${ }^{\ddagger}$, B. Lehmann ${ }^{\S}$ and U. Michel ${ }^{\Uparrow}$ \\ German Aerospace Center, Institute of Propulsion Technology, Turbulence Research Branch
}

Indirect combustion noise is investigated experimentally and numerically. This noise is generated in the outlet nozzle of combustion chambers if the entropy of the medium is nonuniform, which is the case in the exhaust of combustors. The contribution to the total noise emission of aeroengine combustors is not known. A test rig for the experimental investigation of this noise emission in the presence of swirl is first described. The indirect noise is generated in an exchangeable convergent-divergent nozzle at the exit of the combustor. The noise radiation is studied in a circular exhaust pipe with probe microphones using a radial mode analysis of the microphone signals. First results of the measured sound fields are reported. The experimental situation will be studied numerically with a $4^{\text {th }}$ order accurate CAA-method, which is first validated with theoretical results of the literature for the cases of a compact nozzle or diffuser and incoming entropy and sound waves in a one-dimensional mean flow. The agreement with the results of sound generation due to incoming entropy waves and the sound reflection and transmission for incoming sound waves is very good. The method is then applied to the more realistic cases of non-compact nozzles and it is found that the amplitudes of the generated waves are substantially smaller in comparison to the one-dimensional theory. The sound generation of real cases like a swirling hot-spot and entropy waves in a one-dimensional flow through a convergent-divergent nozzle as well as plain entropy waves in the swirl flow of the experimental setup are finally studied and the noise emission is computed.

$\Delta l=$ length of the nozzle

$\gamma=$ ratio of the specific heats in a gas

$\varrho^{\prime}=$ density perturbation

$\varphi=$ angular coordinate

$\omega=$ angular frequency

$M=$ Mach number

$R_{a}=$ Reflection coefficient

\section{Nomenclature}

$R=$ Radius of the inlet duct
$T_{a}=$ Transmission coefficient
$a=$ local speed of sound
$p^{\prime}=$ pressure perturbation
$r \quad=$ radial coordinate
$x=$ axial coordinate
Subscript

$1=$ section upstream of the nozzle

$2=$ section downstream of the nozzle

$+\quad=$ downstream propagating

$-\quad=$ upstream propagating

$s \quad=$ entropy mode wave

\footnotetext{
${ }^{*}$ Research Assistant, Müller-Breslau-Str. 8, 10623 Berlin, Germany, AIAA Student Member

${ }^{\dagger}$ Professor, Müller-Breslau-Str. 8, 10623 Berlin, Germany, AIAA Member

${ }^{\ddagger}$ Research Assistant, Müller-Breslau-Str. 8, 10623 Berlin, Germany

§Scientist, Müller-Breslau-Str. 8, 10623 Berlin, Germany

๑Senior Scientist, Müller-Breslau-Str. 8, 10623 Berlin, Germany, AIAA Member
} 


\section{Introduction}

Combustion noise has become an increasingly important contribution to aeroengine noise, especially during the landing approach of modern aircraft. This experience is a consequence of the successes in decreasing jet mixing noise and fan noise of modern aero-engines. The total noise emitted by a combustion chamber consists of direct and indirect combustion noise. Only the direct combustion noise is related to the combustion process. The indirect combustion noise is generated when fluid with a nonuniform entropy distribution is accelerated in or convected through the nozzle lo-

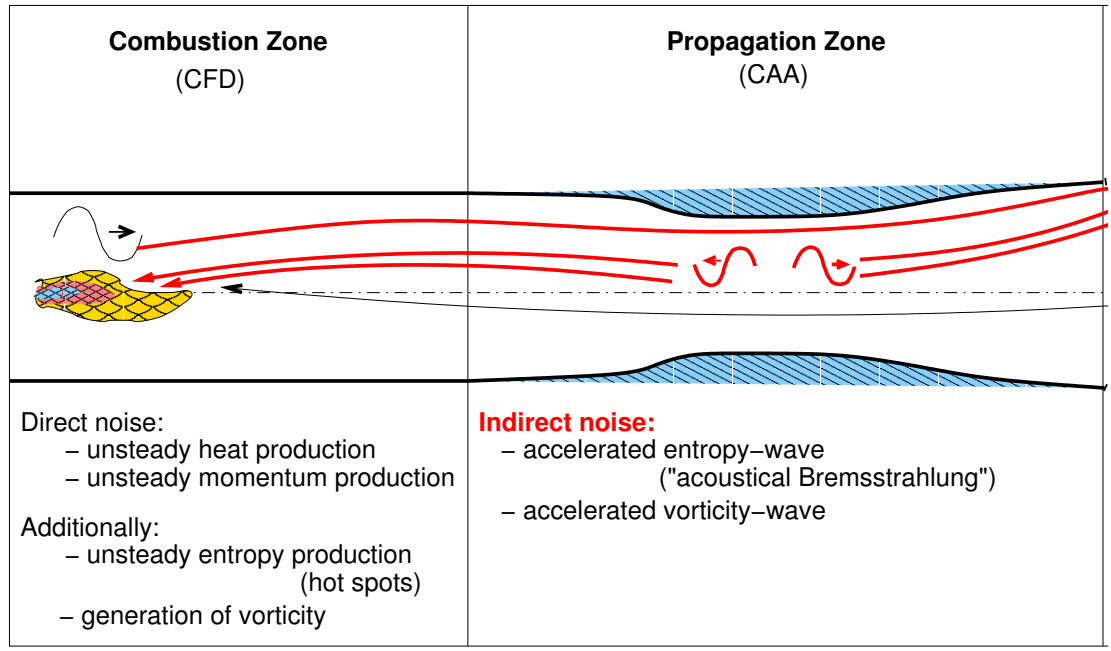

Figure 1. Sources of sound in a combustion chamber with outlet nozzle cated at the downstream end of the combustion chamber (Fig. 1). The accelerated or decelerated hot spot radiates sound due to a fluctuating mass flux. This sound source is fully described by the Computational Aeroacoustic (CAA) approach presented to address the noise propagation and radiation in the outlet section. In gas turbines, the inlet guide vanes for the first turbine stage serve as nozzle for the combustion chamber. The flow in this nozzle is choked in aero-engines in practically all relevant operating conditions. The relation of indirect combustion noise to direct combustion noise is not known. This work therefore addresses the indirect combustion noise and its contribution to the total noise emission of combustion chambers. The basic investigation of the indirect combustion noise is carried out both numerically and experimentally in this work.

\section{Model Experiment}

The setup for the experimental investigation is carefully chosen to replicate combustion flow characteristics of full scale gas turbines while still permitting analysis by experimental means. In order to simplify the numerical approach the system is designed axisymmetrical.

\section{A. Setup}

The experimental rig consists of a cylindrical model combustion chamber made of fused quartz glass. It is driven by a swirled dual air-flow nozzle in order to stabilize the combustion zone. Methane gas is introduced as the fuel between the air streams. The maximum thermal power is $40 \mathrm{~kW}$. The combustion

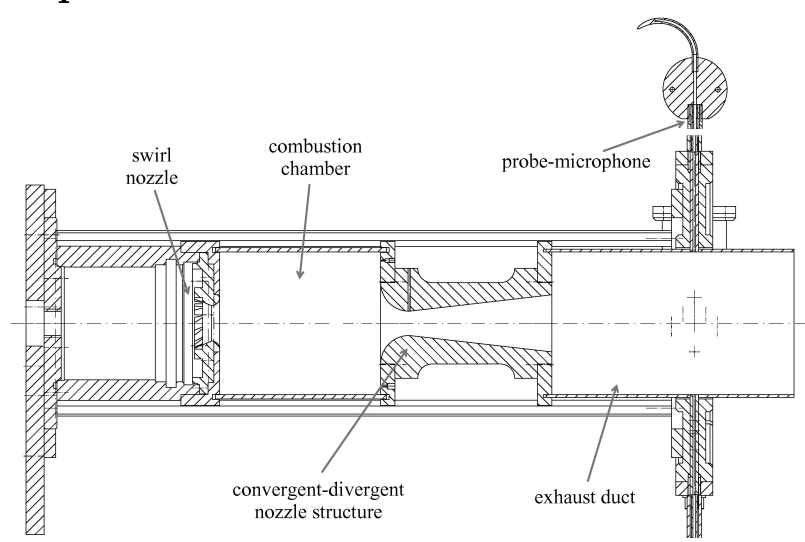

Figure 2. Sketch of the combustion chamber setup used for the model experiments. 
chamber is terminated with a convergent-divergent nozzle structure as shown in Fig. 2. The nozzle shape is exchangeable whereby the throat diameters and with it the outlet Mach numbers are variable.

The nozzle is attached to an exhaust duct with the same diameter as the glass combustion chamber. In order to reduce the impedance jump at the exhaust outlet, an end diffuser is installed. In addition, the diffuser, shown in Fig. 3, is perforated with holes of $2 \mathrm{~mm}$ diameter with increasing perforation density towards the exit. The combustion flow of this facility has been previously investigated under different working conditions due to intense analyses of mean and instantaneous velocities and mean temperature.

\section{B. Flow Field Topology}

In order to achieve a basis for the acoustic analysis and to match the numerical flow simulation the flow field in the combustion chamber is mapped using a three-component Laser-

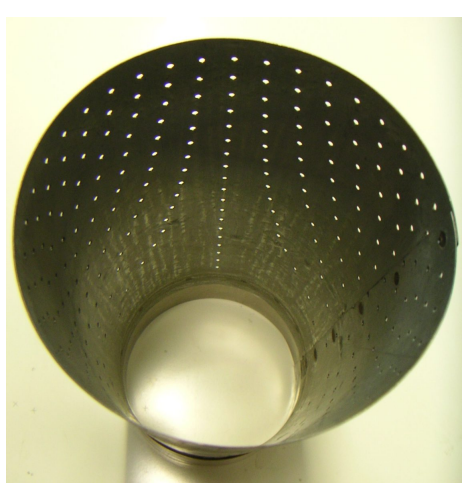

Figure 3. Photo of the perforated end diffuser

Doppler-System. To avoid refraction effects of the laser light a combustion chamber with a square cross section was used. The main feature of the combustion flow field is the strong swirl of the inflow air streams as aforementioned. In Fig. 4 the mean velocity profiles directly after the swirl nozzle exit are plotted. The axial component on the left hand side shows a flame holding recirculation zone on the center axis due to the swirl vortex breakdown. The circumferential component, on the right hand side, has the same order of magnitude and displays two maxima related to the two concentric and co-rotating air flows.
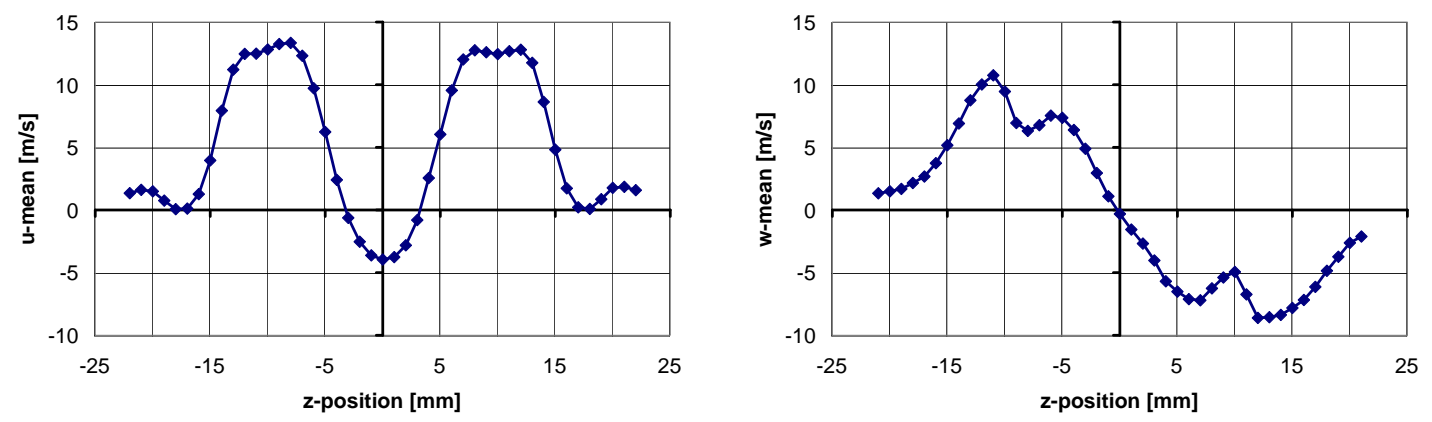

Figure 4. Velocity profiles (LDA-data) of axial (left) and circumferential (right, same scale) component downstream of the swirl nozzle in the combustion zone.

\section{Acoustical Measurement Tools}

Sound measurements in combustion environments make high demands on the acoustic equipment. High temperatures up to $2000 \mathrm{~K}$ and highly corrosive exhaust gases disallow the usage of normal microphone setups in the standard way. To prevent sensor destruction a probe microphone configuration like shown in Fig. 5 is used.

Due to the spatial separation of the measurement location at the combustion chamber wall or exhaust duct and the microphone itself, common 1/4-inch-microphones can be used. The connection between the 
exhaust duct wall and the microphone is realized by a steel tube of $2 \mathrm{~mm}$ inner diameter. For impedance matching and to avoid standing wave effects in the probe tube, this is extended according to the principle of the semi-infinite acoustic duct.

The microphone itself is perpendicular and flush-mounted inside the cylindrical chamber shown in Fig. 5. From the rear end, the probe tube is purged with cooling gas, which also prevents the diaphragm of the microphone from damage by corrosive combustion products. Of course, the phase shift in the collected data due to the propagating delay through the probe tube has to be corrected afterwards.

In the current setup twelve microphones can be installed on the exhaust duct system at three axial and four circumferential positions. From the calibrated time-series, the downstream and upstream propagating acoustical waves are recalculated as sketched in Fig. 6 using an inhouse processing code. ${ }^{1,2}$ In the considered frequency range, the plane wave modes are the only propagating modes.

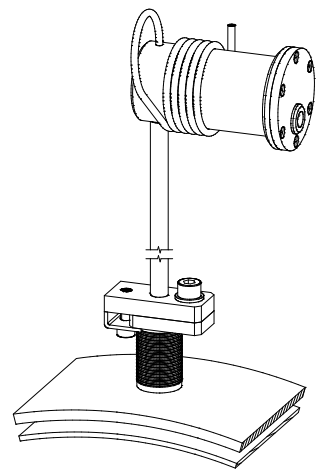

In the first test series Mach numbers between 0.05 and 0.28 in the outlet nozzle have been investigated. The thermal power ranged from 10 to $20 \mathrm{~kW}$. Three probe microphones at the three axial positions were used for the acoustical data acquisition. Figure 7 shows the separation of the downstream and upstream propagating acoustical power versus the frequency.

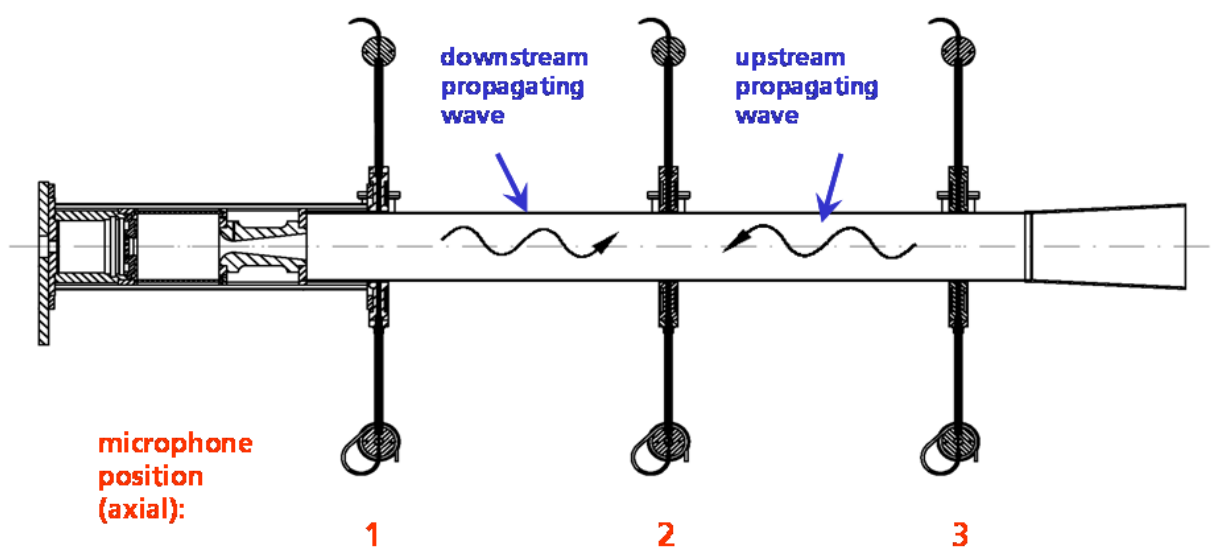

Figure 6. Separation of upstream and downstream propagating plane waves in the exhaust duct via mode analysis.

The remarkable peak at about $100 \mathrm{~Hz}$ is a result of self-excited oscillations of the combustion system at an operational power of $10 \mathrm{~kW}$. At this peak frequency the upstream running waves on the right hand side show an about $7 \mathrm{~dB}$ lower amplitude in acoustical power than the downstream running waves on the left. This determines the power reflection coefficient to less than 0.2 which is much smaller than the reflection coefficient of an open duct end. The expected wavelength of the entropy perturbation in the wake of the flame would be of the order of the nozzle length, while the typical length scales of the turbulent mixing processes in the combustion chamber are in the order of $0.5 \%$ of the nozzle length with a typical frequency of $1250 \mathrm{~Hz}$. The present experimental results show that the noise contribution of the turbulent mixing processes in the mentioned frequency range play a minor role to the total noise emitted by the combustion chamber system, and do not effect the low frequency noise emission investigated here. 

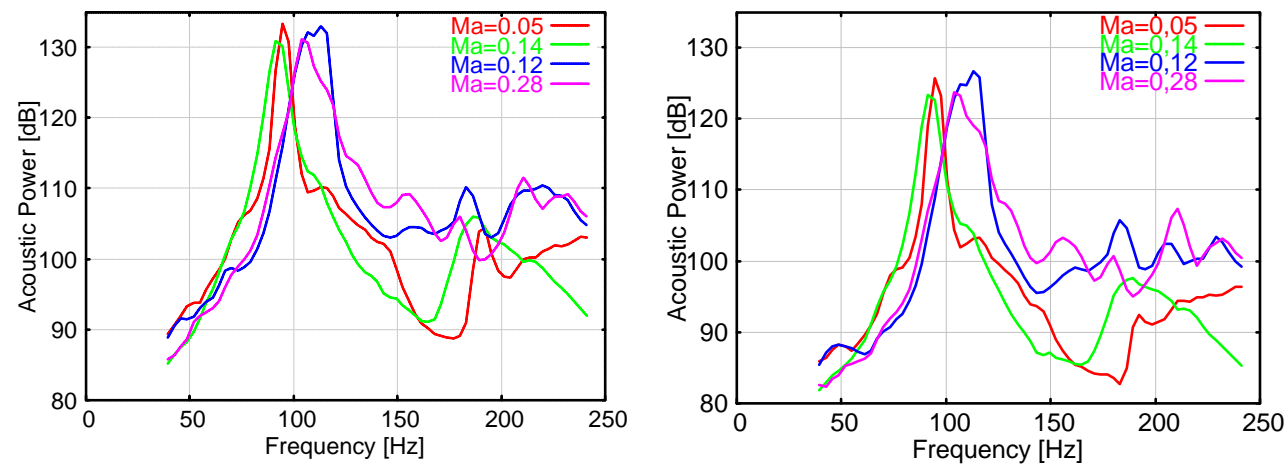

Figure 7. Acoustical power spectra of downstream (left) and upstream (right) propagating waves.

\section{Numerical method}

The full Navier-Stokes equations describe the wave propagation in the combustion chamber and the outlet section. However, the numerical methods capable of solving these equations produce high computational costs. Even with modern computer power, the resolution required for the convection of density fluctuations in the slow combustion chamber flow is often beyond the scope of problems to be solved in a reasonable time. Entropy fluctuations are usually excited at higher amplitudes for low frequencies. Neglecting the heat transfer and the turbulent mixing processes, a CAA approach, as is established for interior noise propagation in aero-engines inlets, ${ }^{3}$ is suitable for description of the propagation of entropy waves and their sound radiation.

The linearized Euler equations decomposed for each azimuthal mode are specified in a cylindrical coordinate system for an axisymmetric non-isentropic swirling mean flow. The solution of these equations is obtained by a CAA method. The spatial discretization of this method is based on the optimized fourth order Dispersion-Relation-Preserving scheme. ${ }^{4}$ The time stepping utilizes alternating optimized fourth order six stage and five stage Runge-Kutta-schemes ${ }^{5}$ in $2 \mathrm{~N}$ storage form. ${ }^{6}$ In order to suppress short wavelength components, a selective artificial damping ${ }^{7}$ is employed.

\section{A. Validation}

The first step in the development of a numerical method based on the fully non-isentropic linearized Euler equations, is to show that it is able to reproduce theoretically predicted relative sound radiation $p^{\prime} / \varrho_{s}^{\prime}$, reflection $R$ and transmission coefficients $T$ in a nozzle flow from a given initial entropy fluctuation $\varrho_{s}^{\prime}$ or sound wave $p_{1+}^{\prime}$. As a theoretical benchmark, we choose the compact nozzle considerations of Marble \& Candel. ${ }^{8}$ This approach fits the requirement of compressible, subsonic flows for validation with entropy and acoustic wave propagation best. The theory is however limited to compact simple convergent or divergent nozzle configurations where the

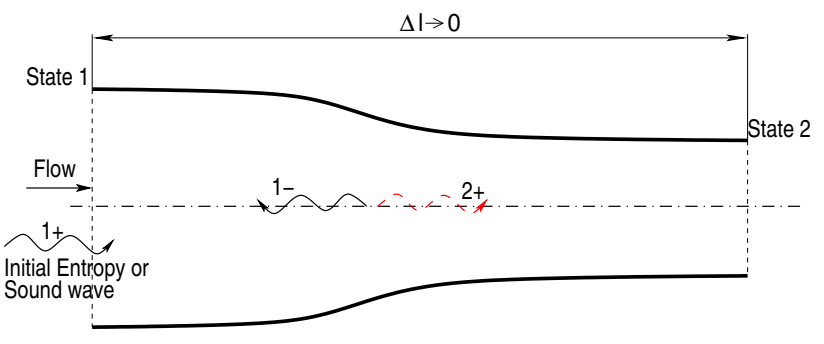

Figure 8. Sketch of the nozzle setup used for the validation experiments wavelength of a perturbation constant in the $r-\varphi$ plane is much larger than the nozzle length $\Delta l$. The critical condition for the compactness is given by the non-isentropic density fluctuation $\varrho_{s}^{\prime}$ convected with the flow speed and the upstream traveling sound wave 
$p_{1-}^{\prime}$ respectively. The numerical discretization is determined by $\Delta l$, which has to be resolved by at least 10 points. The theoretical result for the nozzle shown in Fig 8, based on the assumption of a one-dimensional compressible mean flow, is restated from Marble \& $\mathrm{Candel}^{8}$ in Table 1 as function of the inlet Mach-number $M_{1}$, the outlet Mach-number $M_{2}$, the ratio of the specific heats $\gamma$ and the inlet speed of sound $a_{1}$.

The free parameters $\left(M_{1}, M_{2}, a_{1}\right.$ and $\left.\gamma\right)$ for the theoretical comparison setup in a isentropic potential mean flow are determined by the contraction ratio of the nozzle or diffuser (reversed nozzle) and the inlet Mach number $M_{1}$. In order to validate the result obtained from the CAA-method over a wide range of subsonic configurations, the geometry as well as the inlet Mach number are varied. The CAA-method was initially developed for the inlet and is actually 3D axisymmetric, while the theoretical setup is described two dimensionally by the method. The generic nozzle geometry is described by a sine function shape. The input is a plain harmonic non-isentropic density perturbation for the results given in Fig. 9 - 10, and plain acoustic waves for Fig. 11. For the low frequencies considered in the validation setup, only plane acoustic waves are cut-on in the observed configurations.

Table 1. Collection of the theoretical assumed plain wave propagation coefficients in a one-dimensional compressible flow (compare Marble \& $\mathrm{Candel}^{8}$ )

\begin{tabular}{c|l} 
input & transfer function \\
\hline \hline & $\frac{p_{1-}^{\prime}}{\varrho_{s}^{\prime}}=-\left(\frac{M_{2}-M_{1}}{1-M_{1}}\right)\left(\frac{\frac{1}{2} M_{1}}{1+\frac{1}{2}(\gamma-1) M_{1} M_{2}}\right) a_{1}^{2}$ \\
$\varrho_{s}^{\prime}$ & $\frac{p_{2+}^{\prime}}{\varrho_{s}^{\prime}}=\left(\frac{M_{2}-M_{1}}{1+M_{2}}\right)\left(\frac{\frac{1}{2} M_{2}}{1+\frac{1}{2}(\gamma-1) M_{1} M_{2}}\right) a_{1}^{2}\left(\frac{1+\frac{1}{2}(\gamma-1) M_{1}^{2}}{1+\frac{1}{2}(\gamma-1) M_{2}^{2}}\right)^{\frac{\gamma}{\gamma-1}}$ \\
\hline$p_{1+}^{\prime}$ & $R_{a}:=\frac{p_{1-}^{\prime}}{p_{1+}^{\prime}}=\left(\frac{1+M_{1}}{M_{1}+M_{2}}\right)\left(\frac{M_{2}-M_{1}}{1-M_{1}}\right)\left(\frac{1-\frac{1}{2}(\gamma-1) M_{1} M_{2}}{1+\frac{1}{2}(\gamma-1) M_{1} M_{2}}\right)$ \\
\hline$p_{1+}^{\prime}$ & $T_{a}\left(\frac{1+M_{1}}{M_{1}+M_{2}}\right)\left(\frac{2 M_{2}}{1+M_{2}}\right)\left(\frac{1+\frac{1}{2}(\gamma-1) M_{2}^{2}}{1+\frac{1}{2}(\gamma-1) M_{1} M_{2}}\right)\left(\frac{1+\frac{1}{2}(\gamma-1) M_{1}^{2}}{1+\frac{1}{2}(\gamma-1) M_{2}^{2}}\right)^{\frac{\gamma}{\gamma-1}}$ \\
\hline
\end{tabular}

\section{Sound radiation by entropy waves}

The radiation amplitude of acoustic waves caused by a harmonic plane entropy perturbation excited with low frequencies passing different nozzles are given in Fig. 9. The 2D pressure amplitude of the acoustic waves are averaged over the radius and the straight inlet as well as the straight outlet duct, leading to the amplitudes of the plain acoustic wave in these sections. The ratio of radiated waves and initial entropy perturbation is stated non-dimensionally by dividing the ratio by the square of the speed of sound in the inlet. For the diagram, the phase shift between the downstream traveling wave and entropy perturbation is accounted for by the change in sign. The result is very close to the theory. However, the deviation reaches up to $14 \%$ of the theoretically predicted value for the highest contraction ratio. The calculations for the two different frequencies show only small differences up to $1.4 \%$. This validates that the nozzle can be assumed compact for both frequencies.

Figure 10 summarizes the results for multiple diffuser configurations. Different from the nozzles, the upstream traveling acoustic wave is in opposite phase while the downstream excited acoustic wave is in phase with the entropy wave. The deviation reaches $13 \%$ for the upstream radiated wave at the higher 

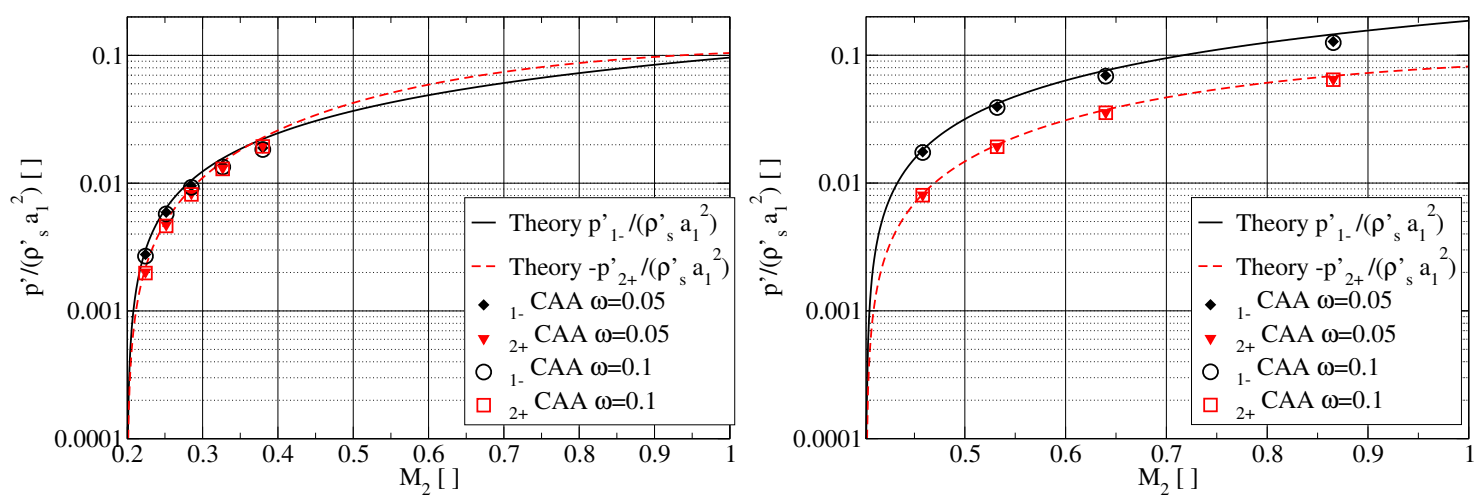

Figure 9. Comparison of the excited relative non-dimensional acoustic pressure amplitude for different compact nozzle configurations driven by an entropy wave at inflow $M_{1}=0.2$ (left), $M_{1}=0.4$ (right)
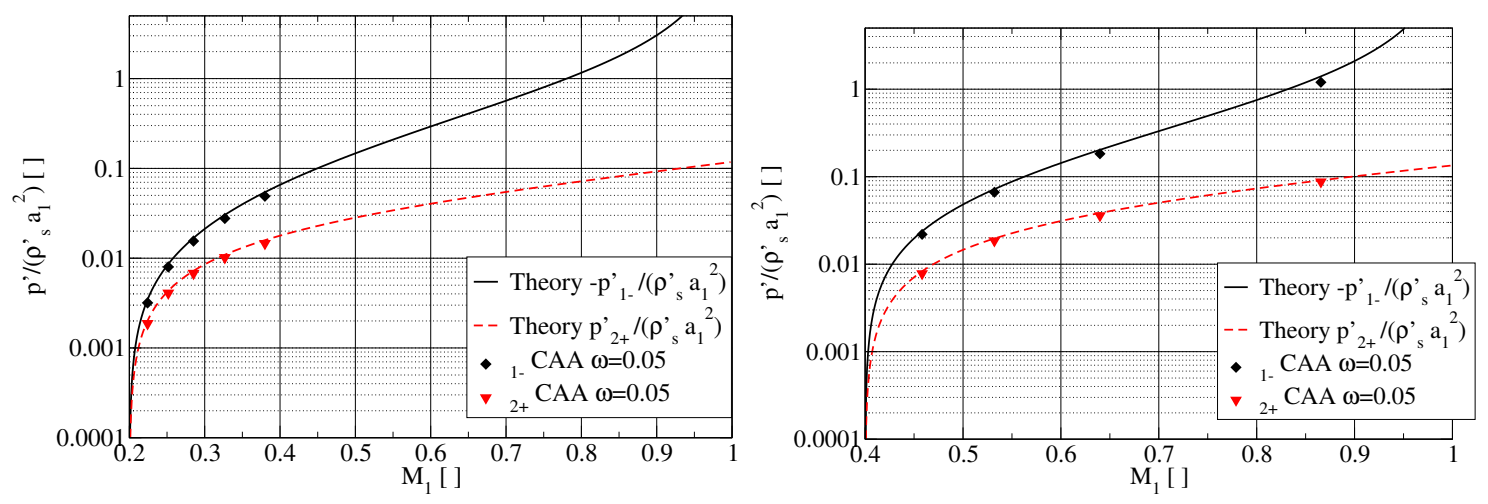

Figure 10. Comparison of the excited relative non-dimensional acoustic pressure amplitude for different compact diffuser configurations driven by an entropy wave $\left(M_{2}=0.2\right.$ left, $M_{2}=0.4$ right)
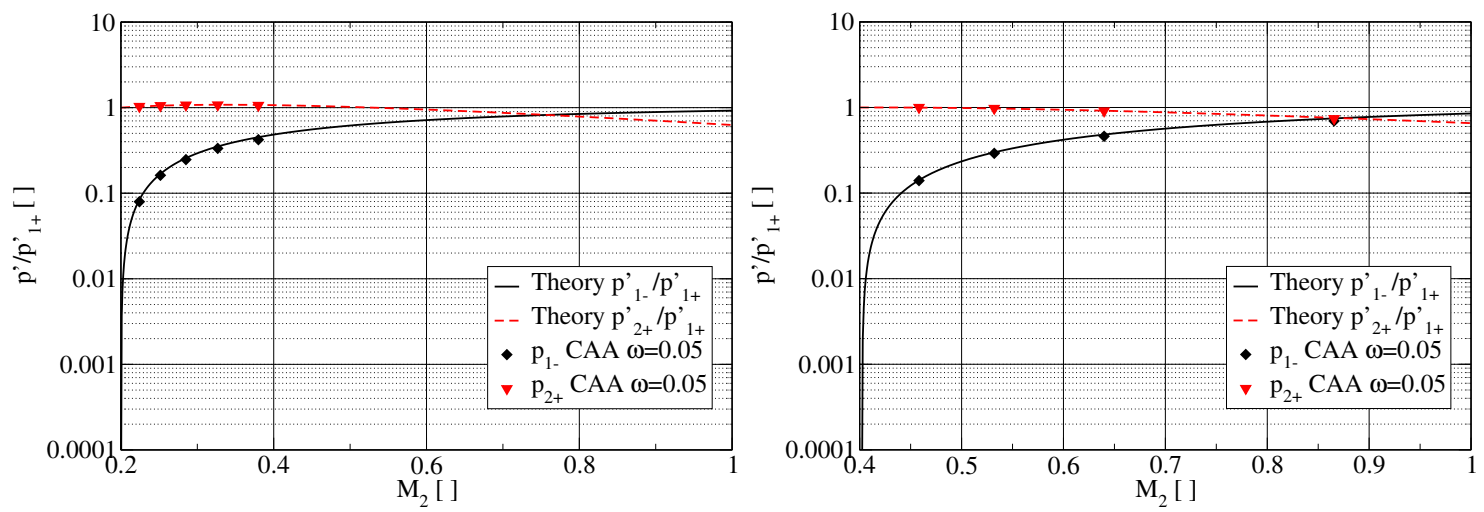

Figure 11. Comparison of the excited relative acoustic pressure amplitude for different compact diffuser configurations driven by an initial sound wave $\left(M_{2}=0.2\right.$ left, $M_{2}=0.4$ right $)$ 
contracted nozzles.

\section{Acoustic wave reflection and transmission}

Figure 11 gives an example for the validation on the acoustic wave propagation. In order to model the propagation of sound waves excited by unsteady combustion, the plain waves are inserted at the upstream end of the nozzle. Due to the acceleration in the nozzle, the acoustic waves are partly reflected and only a fraction of acoustic energy is transmitted downstream through the nozzle. The reflected wave is overpredicted by a maximum of $6 \%$ in the case of $M a=0.2$. The maximum deviation is reached for the highest contraction at $M a=0.4$ with about $6 \%$ underprediction of the pressure amplitude in the inlet duct by the CAA-method.

\section{Discussion of the validation}

The result of a two dimensional calculation is compared to a 1D theory, which does not account for the radial mean flow effects. This difference from the theoretically assumed configuration is in the order of the observed deviation, as it is shown in Fig. 12. The radial velocity effects may explain the difference from the theory observed in the calculations above. However in general, the result given here has shown that the entropy wave propagation and the sound radiation by simple nozzles is described according to the theory by the current CAA-method. The method is applicable to the low frequency propagation of entropy waves through a nozzle.

\section{B. Some parametric studies on entropy waves}

In order to show the capabilities of the presented approach, some parameters are varied away from the theoretically considerable cases.

\section{Non-compact nozzle driven by plain entropy waves}

To show what happens if the nozzle is non-compact, the frequency driving the entropy wave excitation is varied. The higher angular frequencies in Fig. 13 represent a lower wavelength of the harmonic non-isentropic entropy perturbation. At the angular frequency of $\omega=1.34$ the wavelength of the entropy perturbation in the inflow tube is in the order of the nozzle length. The nozzle is not compact. For comparison the theoretical result for compact nozzles is also shown in Fig. 13. The first radial mode can propagate in the outlet duct for $\omega>2.44$, the second for $\omega>4.47$. In order to resolve the wavelength of the higher frequency entropy waves the resolution of the grid

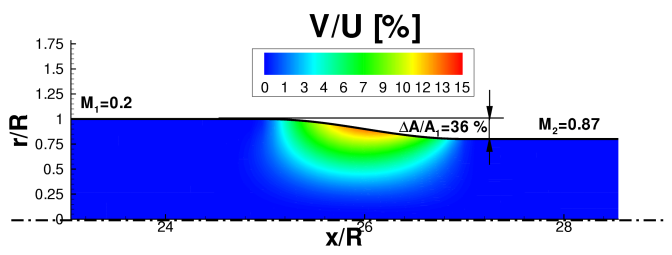

Figure 12. Ratio of the radial velocity and the axial velocity in the contracting nozzle

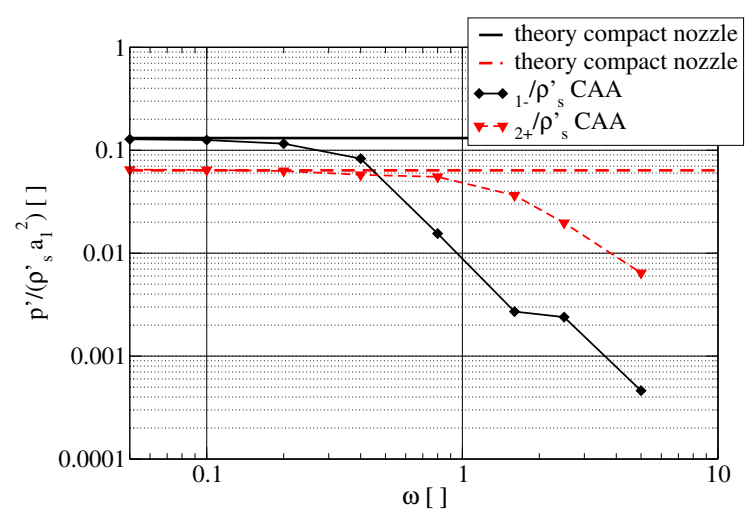

Figure 13. Variation of the frequency of the excited entropy wave for a highly contracted nozzle configuration $\left(M_{1}=0.4, M_{2}=0.86\right)$ has been increased by a factor of ten compared to the mesh used for the validation. The spatial resolution obtained for these cases is above 20 points per wavelength (ppw) for the entropy waves. The acoustic waves are therefore resolved at least by $30 \mathrm{ppw}$ for the upstream plain acoustic waves. The amplitude of the excited plain acoustic waves decrease with the decrease of the wavelength of the entropy waves. Especially the backward traveling waves show a strong decrease in 
amplitude (Fig. 13). The accelerated non-isentropic density perturbation is compressed or expanded, when accelerating to the flow state in the outlet duct of the generic nozzle, but the radiated sound waves are not in phase for the non-compact nozzle configurations. The upstream traveling waves have lower wavelength and are therefore more strongly effected by the phase shift.

\section{Hot spot revolving in the duct}

In real applications the shape of the density fluctuation would differ from the plane wave. For a swirling, non-premixed flame a perturbation moving periodically around the axis of the flame is another probable form. In order to understand the mechanisms, we assume a Bessel function-like distribution with a maximum at the outer wall for the non-isentropic perturbation in density, and input by the flame. The observation from such an entropy wave exciting a cut-off mode in Fig. 14, is that the curved parts of the nozzle contribute with higher amplitude sound radiation, while the longer conical part between excites much lower amplitude sound waves for this mode.

\section{Complex convergent-divergent subsonic nozzles}

Fig. 15 demonstrates one preliminary result based on the swirling flow behind the swirl stabilized nonpremixed burner. The mean flow is based on a RANS calculation. The entropy fluctuation is introduced by a $1 \%$ harmonic perturbation of the mean density excited in the combustion chamber. The swirl leads to a non-detached flow in the divergent part of the model nozzle. In order to briefly preinvestigate a more realistic configuration, a more complex convergent-divergent generic nozzle contour is generated based on sine functions of different length. The mean flow is assumed to be a one-dimensional

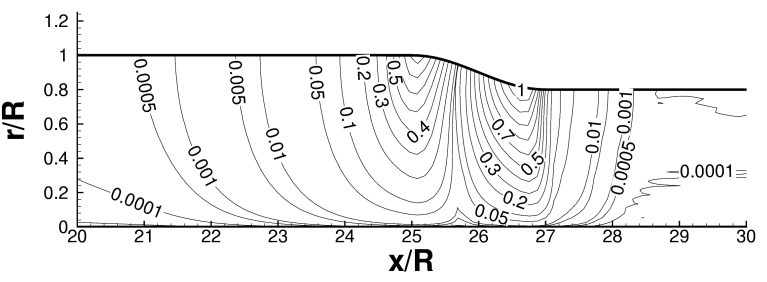

Figure 14. Attenuation of sound pressure excited in the cut-off mode $m=1, n=1\left(M_{1}=0.4, M_{2}=0.86, \omega=0.2\right)$ potential flow, as in the examples given before. The inflow Mach number is $M_{1}=0.035$, and the nozzle contraction is varied from no contraction to the smallest area of $25 \%$ of the inlet duct. The results summarized in Fig. 16 illustrate the effect of the contraction ratio on the noise radiation. The divergent section of the nozzle is twice as long as the convergent part, and case a) is the validation case for a non-contracted nozzle radiating no sound from an initial entropy wave. The sound pressure levels obtained non-isentropically by a density fluctuation of $1 \%$ from the mean state, reach $72 \mathrm{~dB}$ in the inlet and $73 \mathrm{~dB}$ in the outlet for case b), and $93 \mathrm{~dB}$ for the higher contracted nozzle case c) in inlet and outlet. The sound pressure levels are very similar in the inlet and outlet section. This is explained by the long sound waves reflected and transmitted at levels next to one (compare Fig. 11) as they are propagating through the compact nozzle. The noise radiation of the nozzle structure increases with the contraction and the acceleration of the flow. 


\section{Conclusion}

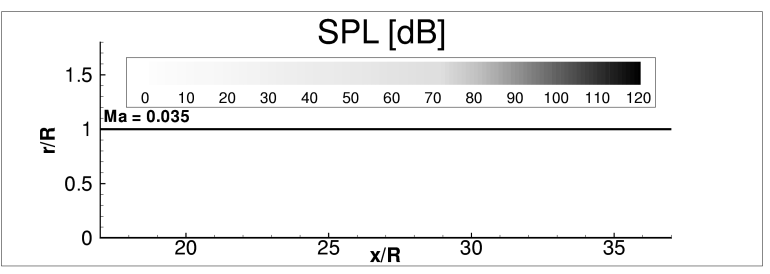

a) $\Delta r / R=0.0$

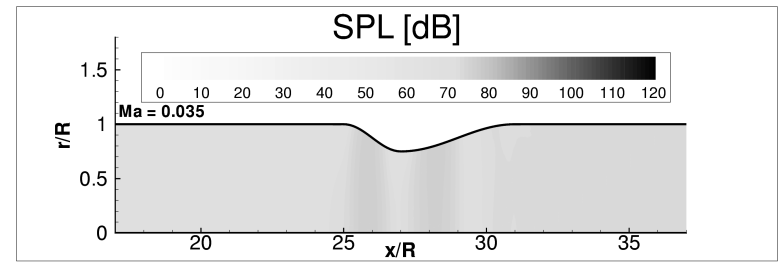

b) $\Delta r / R=0.25$

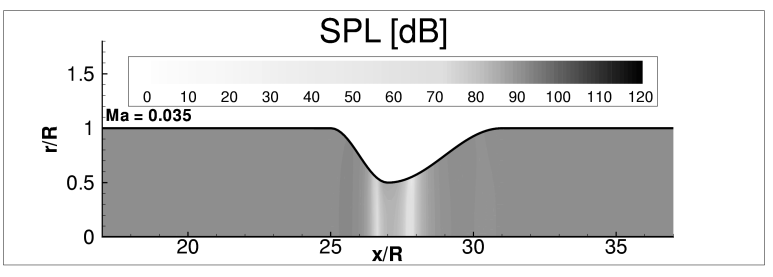

c) $\Delta r / R=0.5$
It is demonstrated that the sound generation due to entropy nonuniformities in the flow through the nozzle at the end of a combustion chamber can be determined numerically with a CAA method. The real situation with non-compact nozzles as well as with a high swirl in the mean flow can be treated. It is shown that the noise generation mechanism in the nozzle yields acoustic waves that travel in both directions, downstream toward the turbines in aeroengines as well as upstream back into the combustor. The latter can cause combustion instabilities, which yield rumble. The experimental tests have demonstrated that it is possible to determine the acoustic power of the sound field in the exhaust pipe through radial mode analysis. A termination of the exhaust pipe with a small reflection coefficient increases the robustness of the results. The sound related to entropy nonuniformities in the flow will be investigated using temperature signals from upstream the nozzle throat. The experimental situation will be duplicated in the numerical procedure as a next step.

\section{Acknowledgments}

The authors gratefully acknowledge the financial support of the German Research Foundation (DFG) through the Research Unit FOR 486 "Combustion Noise".

Figure 16. Convergent-divergent nozzle configurations driven by plain entropy waves $\left(M_{1}=0.035, \omega=1.0\right)$

\section{References}

\footnotetext{
${ }^{1}$ Enghardt, L., Zhang, Y., and Neise, W., "Experimental verification of a radial mode analysis technique using wall-flush mounted sensors," 137th Meeting of the Acoustical Society of America, Berlin, October 1999, pp. 15-19.

${ }^{2}$ Maier, R., Zillmann, J., Roure, A., Winninger, M., Enghardt, L., Tapken, U., Neise, W., Antoine, H., and Bouty, E., "Active Control of Fan Tone Noise from Aircraft Engines," 7th AIAA/CEAS Aeroacoustics Conference, No. 2001-2220, Maastricht, 2001.

${ }^{3} \mathrm{Li}$, X., Schemel, C., Michel, U., and Thiele, F., "On the Azimuthal Mode Propagation in Axisymmetric Duct Flows," No. 2002-2521, 2002.

${ }^{4}$ Tam, C. K. W. and Webb, C., "Dispersion-Relation-Preserving Finite Difference Schemes for Computational Aeroacoustics," J. Comp Phys., Vol. 107, No. 2, August 1993, pp. 262-281.

${ }^{5}$ Hu, F. Q., Hussani, M. Y., and Manthey, J. L., "Low-dissipation and Low-dispersion Runge-Kutta Schemes for Computational Acoustics," J. Comput. Phys., Vol. 124, No. 1, 1996, pp. 177-191.

${ }^{6}$ Stanescu, D. and Habashi, W., "2N-storage Low-dissipation and Low-dispersion Runge-Kutta Schemes for Computational Aeroacoustics," J. Comput. Phys., Vol. 143, No. 2, 1998, pp. 674-681.

${ }^{7}$ Tam, C. K. W., Webb, C., and Dong, T. Z., "A Study of Short Wave Components in Computational Aeroacoustics," Journal of Computational Acoustics, Vol. 1, March 1993, pp. 1-30.

${ }^{8}$ Marble, F. and Candel, S., "Acoustic disturbances from gas non-uniformities convected through a nozzle," J. Sound Vib., Vol. 55, No. 2, 1977, pp. 225-243.
}

10 of 10 